\title{
The dualizing spectrum II
}

\author{
JOHN R KLEIN
}

\begin{abstract}
To an inclusion $H \subset G$ of topological groups, we associate a spectrum $D_{H \subset G}$, which coincides with the dualizing spectrum $D_{G}$ of Klein [5] when $H=G$. We also introduce a fibered spectrum analogue.

The main application is to give a purely homotopy theoretic construction of Poincaré embeddings in stable codimension.
\end{abstract}

$55 \mathrm{P} 91 ; 57 \mathrm{P} 10$

\section{Introduction}

An embedding of manifolds $P \subset N$ and a compact tubular neighborhood $D$ of $P$ in $N$ gives rise to a stratification

$$
N=D \cup_{\partial D} C,
$$

where $C$ is the closure of $N-D$. From a homotopy theorist's perspective, it is legitimate to consider a variant of the above in which the manifolds are replaced by Poincaré duality spaces, and equality is replaced by homotopy equivalence. This yields the notion of Poincaré embedding. It turns out that the "normal datum" $\partial D \rightarrow D$ has the homotopy type of a spherical fibration over $P$.

One the early triumphs of surgery theory is the Browder-Casson-Sullivan-Wall theorem. It says that a Poincaré embedding of PL manifolds in codimension at least three can always be lifted to a piecewise linear embedding. Thus the problem of finding manifold embeddings in these codimensions is reduced to a problem in homotopy theory. Even so, at least one golden era maven opined that the resulting homotopy theoretic problems seem "in some respects to be harder than the original geometrical problems" (see Wall [16, p 119]).

Fortunately, much of the homotopy theory was tractable, although it took a quarter century to realize it. By the late 1990s, an array of Poincaré embedding results were proved by homotopy theoretic means. For example, see Richter [11], Klein [2; 5; 6; 7], Lambrechts and Stanley [8] and Lambrechts, Stanley and Vandembroucq [9]; some of these works address a program begun by B Williams in the late 1970s [17; 18]. 
Actually, the first Poincare embedding result in the literature predates the BrowderCasson-Sullivan-Wall theorem: Spivak's thesis, somewhat reformulated, delivered Poincaré embeddings in Euclidean space in large codimension and showed that such embeddings are unique up to concordance [13]. The normal datum in this case is called the Spivak fibration.

Spivak's results were proved manifold theoretically. A few years ago, Bill Dwyer and the author (independently) observed how the Spivak fibration could be obtained by an entirely homotopy theoretic procedure [5]. ${ }^{1}$

The main purpose of this paper is to extend some ideas of [5], with the aim of giving a homotopy theoretic proof of the following theorem:

Theorem A (Existence) Let $f: P \rightarrow N$ be a map of finitely dominated Poincaré duality spaces, where $P$ is without boundary. Then there is an integer $j>0$ and a Poincaré embedding of the composite map

$$
f_{j}: P \stackrel{f}{\longrightarrow} N \stackrel{\subset}{\longrightarrow} N \times D^{j} .
$$

Remark 1.1 In [2], we used this result as the basis step of a downward induction on codimension to produce Poincaré embeddings in the range where, roughly, "connectivity exceeds generic double point dimension." The proof we gave in Section 3 of that paper used a manifold thickening of the ambient space together with transversality.

In [2] we considered the more general situation in which $P$ is merely a finite complex. The methods of the current paper do extend to this more general case, but for reasons of exposition we decided to retain the assumption that $P$ satisfies Poincaré duality.

We shall also prove the following uniqueness result:

Theorem B (Uniqueness) If $j$ is sufficiently large, then any two Poincaré embeddings of $f_{j}: P \rightarrow N \times D^{j}$ are concordant.

Outline Section 2 is language. In Section 3 we recall the dualizing spectrum of a topological group and extend it to inclusions. In Section 4 we prove Theorem A in the connected case. In Section 5 we describe a fibered spectrum which has the homotopy type of the unreduced Borel construction of the dualizing spectrum. In Section 6 we complete the proof of Theorem A. In Section 7 we prove Theorem B. In Section 8 we present a type of fiberwise duality, due to Bill Richter, which used in the proof of Theorem B.

\footnotetext{
${ }^{1}$ The construction has recently been used to prove that finite loop spaces have the homotopy type of smooth manifolds, partially settling a question of Browder [1].
} 
Acknowledgements I am indebted to Bill Richter for explaining to me the duality theory described in Section 8. The author is partially supported by the NSF.

\section{Language}

This section somewhat abridged. The reader is directed to [5, Section 2] for a more complete treatment.

All spaces below will be compactly generated. A weak equivalence of spaces means a weak homotopy equivalence. A space is homotopy finite it is weak equivalent to a finite cell complex. It is finitely dominated if it is a homotopically a retract of a finite cell complex.

\section{Poincaré spaces}

A finitely dominated space $X$ is a Poincaré duality space of (formal) dimension $d$ if there exists a bundle of coefficients $\mathcal{L}$ which is locally isomorphic to $\mathbb{Z}$, and a fundamental class $[X] \in H_{d}(X ; \mathcal{L})$ such that the associated cap product homomorphism

$$
\cap[X]: H^{*}(X ; M) \rightarrow H_{d-*}(X ; \mathcal{L} \otimes M)
$$

is an isomorphism in all degrees. Here, $M$ denotes any bundle of coefficients (cf Wall [15] and Klein [4]).

Similarly, one has the definition of Poincaré space $X$ with boundary $\partial X$ (also called a Poincaré pair $(X, \partial X))$. Here, one assumes both $X$ and $\partial X$ are finitely dominated and there is a fundamental class $[X] \in H_{d}(X, \partial X ; \mathcal{L})$ such that

$$
\cap[X]: H^{*}(X ; M) \rightarrow H_{d-*}(X, \partial X ; \mathcal{L} \otimes M)
$$

is an isomorphism. Additionally, if $[\partial X]$ is the image of $[X]$ under the boundary homomorphism $H_{d}(X, \partial X ; \mathcal{L}) \rightarrow H_{d-1}\left(\partial X ; \mathcal{L}_{\mid \partial X}\right)$, one also requires

$$
\left(\mathcal{L}_{\mid \partial X},[\partial X]\right)
$$

to equip $\partial X$ with the structure of a Poincaré space.

The above definition makes sense even if the map $\partial X \rightarrow X$ fails to be an inclusion, since one can always take a mapping cylinder to convert the map into a cofibration. We shall sometimes work in this more general context.

Algebraic 83 Geometric Topology, Volume 7 (2007) 


\section{Poincaré embeddings}

Let $P^{p}$ and $N^{n}$ be a Poincaré spaces of respective dimensions $p$ and $n$, with $\partial P=\varnothing$ (but where $N$ is possibly with boundary) and with $p \leq n-1$. A Poincaré embedding of a map $f: P \rightarrow N$ is a commutative diagram

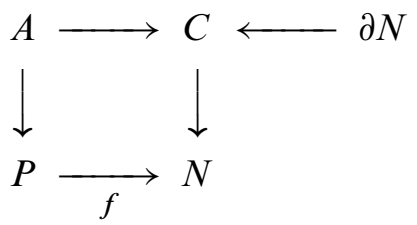

in which the following conditions hold.

- The square in the diagram is a homotopy pushout.

- The map $A \rightarrow P$ has the homotopy type of a $(n-p-1)$-spherical fibration over $P$ (in particular its mapping cylinder $\bar{P}$ is a Poincaré space of dimension $n$ with boundary $A$ ).

- The composite $\partial N \rightarrow C \rightarrow N$ is the inclusion.

- The image of a fundamental class under the composite

$$
H_{n}(N, \partial N ; \mathcal{L}) \rightarrow H_{n}(N, C ; \mathcal{L}) \cong H_{n}\left(P, A ; \mathcal{L}_{\mid P}\right)
$$

equips $P$ with the structure of a Poincare space with boundary $A$. Similarly, the image of a fundamental class under

$$
H_{n}(N, \partial N ; \mathcal{L}) \rightarrow H_{n}(N, P \amalg \partial N ; \mathcal{L}) \cong H_{n}\left(C, A \amalg \partial N ; \mathcal{L}_{\mid C}\right)
$$

equips $C$ with the structure of a Poincare space with boundary $A \amalg \partial N$.

We are not necessarily assuming in the above that $A \rightarrow P$ and $A \amalg \partial N \rightarrow C$ are inclusions. However, we are abusing notation slightly when writing $H_{n}(N, C ; \mathcal{L})$; the reader should substitute the appropriate mapping cylinder in such cases.

Roughly, a concordance of two Poincaré embeddings of $f$, is a map of their associated diagrams which is a weak equivalence at each space of the diagram and which moreover, is the identity at $P, N$ and $\partial N$. We say two embeddings of $f$ are concordant if there is a finite chain of concordances connecting them (see Klein [7] for a more detailed definition). 


\section{Spectra}

A spectrum $X$ is a collection of based spaces $\left\{X_{i}\right\}_{i \in \mathbb{N}}$ together with based maps $\Sigma X_{i} \rightarrow X_{i+1}$ where $\Sigma X_{i}$ denotes the reduced suspension of $X_{i}$. A map of spectra $X \rightarrow Y$ consists of maps $X_{i} \rightarrow Y_{i}$ which are compatible with the structure maps.

Let $G$ be a topological group. For technical reasons, we assume that the underlying space of $G$ is the retract of a cell complex. A (naive) $G$-spectrum consists of a spectrum $X$ such that each $X_{i}$ is a based (left) $G$-space and each structure map $\Sigma X_{i} \rightarrow X_{i+1}$ is equivariant, where it is understood that $G$ acts trivially on the suspension coordinate of $\Sigma X_{i}$. A map of $G$-spectra is a map of spectra that is compatible with the $G$-action. A weak equivalence of $G$-spectra is a morphism inducing an isomorphism on homotopy groups in every degree.

Schwede has shown that $G$-spectra form a Quillen model category with the above notion of weak equivalence [12, Section 2]. For reasons of space, we shall not describe the entire structure, but we will describe the fibrant and cofibrant objects arising from Schwede's model structure. A $G$-spectrum $X$ is fibrant if it is an $\Omega$-spectrum, meaning that the adjoint maps $X_{j} \rightarrow \Omega X_{j+1}$ are weak equivalences for all $j$. It is cofibrant if each $X_{j}$ is built up from a point by attaching free $G$-cells and each structure map $\Sigma X_{j} \rightarrow X_{j+1}$ is obtained up to isomorphism by attaching free cells to $\Sigma X_{j}$. We also consider retracts of such an $X$ to be cofibrant.

The suspension spectrum $\Sigma^{\infty} X$ of a based $G$-space $X$ has $j$-th space $Q\left(S^{j} \wedge X\right)$, where $Q=\Omega^{\infty} \Sigma^{\infty}$ is the stable homotopy functor.

If $X$ is a $G$-spectrum then the homotopy orbit spectrum $X_{h G}$ is the (nonequivariant) spectrum given by

$$
X \wedge_{G}\left(E G_{+}\right),
$$

where $E G$ is the free contractible $G$-space (arising from the bar construction), and $E G_{+}$is the result of adding a basepoint to $E G$. This spectrum has $j$-th space $X_{j} \wedge_{G}\left(E G_{+}\right)$.

The homotopy fixed point spectrum $X^{h G}$ is given by

$$
F\left(E G_{+}, X\right)^{G} .
$$

This is the spectrum whose $j$-th space is the equivariant function space of based maps $E G_{+} \rightarrow X_{j}$. Taking homotopy fixed points is homotopy invariant when $X$ is fibrant.

As in [5], we use naive versions of the smash product (which are associative, unital and commutative up to homotopy).

Algebraic 83 Geometric Topology, Volume 7 (2007) 


\section{The dualizing spectrum of an inclusion}

Let $G$ be a topological group. The group ring of $G$ over the sphere spectrum is the $(G \times G)$-spectrum

$$
S^{0}[G]
$$

which is the suspension spectrum of $G_{+}$. The action of the left copy of $G$ in $G \times G$ on $S^{0}[G]$ is given by left multiplication. The action of the right copy is given by right multiplication composed with inversion $\left(g \mapsto g^{-1}\right)$.

In [5], the dualizing spectrum of $G$

$$
D_{G}=S^{0}[G]^{h G}
$$

was defined as the homotopy fixed points of the left copy of $G$ acting on the group ring. Since the two $G$-actions commute, the right copy of $G$ acts on $D_{G}$ giving it the structure of a $G$-spectrum.

If $h: H \rightarrow G$ is a homomorphism, then $S^{0}[G]$ inherits an $(H \times G)$-action (restriction of scalars), and we have maps

$$
D_{H} \stackrel{h_{!}}{\longrightarrow} S^{0}[G]^{h H} \stackrel{h^{!}}{\longleftarrow} D_{G} .
$$

The first of these, called pushforward, is induced by the map $S^{0}[H] \rightarrow S^{0}[G]$ arising from $h$. The second, called restriction, is given by regarding a $G$-fixed point as an $H$-fixed point. The pushforward map is $H$-equivariant, and the restriction map is $G$-equivariant.

From this point on, we will only need consider the situation where $h: H \rightarrow G$ is an inclusion.

Definition 3.1 The dualizing spectrum associated with an inclusion $H \subset G$ is the $G$-spectrum

$$
D_{H \subset G}:=S^{0}[G]^{h H} .
$$


Before proceeding further, it will be useful to recall some results from [5].

Theorem 3.2 [5, Corollary 5.1] Assume $B G$ is a finitely dominated space. Then:

- $D_{G}$ is a suspension spectrum, ie there are an integer $j \geq 0$, a finitely dominated, 1 -connected based $G$-space $Y$ and an equivariant weak equivalence

$$
\Sigma^{j} D_{G} \simeq \Sigma^{\infty} Y \text {. }
$$

- If furthermore $B G$ is a Poincaré space (of dimension $d$ ), then $Y$ is unequivariantly weak equivalent to a sphere (of dimension $j-d$ ), and the Spivak normal fibration of $B G$ is given by the Borel construction

$$
Y \rightarrow E G \times_{G} Y \rightarrow B G .
$$

We need a partial extension of Theorem 3.2 to $D_{H \subset G}$.

Proposition 3.3 Assume $B H$ is finitely dominated. Then the first conclusion of Theorem 3.2 holds for $D_{H \subset G}$ (ie it is a suspension spectrum).

Proof The proof of the proposition is basically the same as that of the first part of Theorem 3.2. We will merely sketch the argument.

Since $B H$ is finitely dominated, the homotopy quotient $E H \times_{H} G$ is $G$-finitely dominated (since the Borel construction of $G$ acting on it is identified with $B H$ ). By the equivariant duality theory developed in [3], there is an integer $j \geq 0$, a 1-connected finitely dominated based free $G-\mathrm{CW}$ complex $Y$ and a map

$$
d: S^{j} \rightarrow Y \wedge_{G}\left(E H \times_{H} G\right)_{+}
$$

which satisfies duality, meaning that for all cofibrant and fibrant $G$-spectra $E$, the map $d$ induces an equivalence of mapping spectra

$$
\operatorname{map}\left(\left(E H \times_{H} G\right)_{+}, E\right)^{G} \simeq \operatorname{map}\left(S^{j}, Y \wedge_{G} E\right) .
$$

In particular, if $E$ has a $\left(G \times G^{\prime}\right)$-action, the above equivalence is $G^{\prime}$-equivariant.

By "change of groups," the domain of this equivalence is identified with $E^{h H}$. Setting $E=S^{0}[G]$ (considered as a $(G \times G)$-spectrum) the equivalence of mapping spectra therefore becomes

$$
D_{H \subset G} \simeq S^{-j} \wedge Y,
$$

and it is $G$-equivariant. Applying $j$-fold suspension to both sides completes the proof. 


\section{Proof of Theorem A when $P$ is connected}

A choice of basepoint for $P$ makes $f: P \rightarrow N$ a map of based spaces. If $P$ is connected, then $f(P) \subset N$ is contained in a connected component. It will be enough to produce a Poincaré embedding into that connected component. For this reason, we can assume without loss in generality that $N$ is also connected.

Let $H$ be a topological group model for the loop space $\Omega P$ and $G$ be one for $\Omega N$. Here is one way to get such models: let $S . N$ denote the simplicial total singular complex of $N$, and let $G$. denote its Kan loop group. Define $G$ to be the geometric realization of the underlying simplicial set of $G$. Then $N \simeq B G$. Furthermore, the construction is natural, so we get a homomorphism $H \rightarrow G$ whose classifying map $B H \rightarrow B G$ is identified with $f$.

As it stands, the homomorphism $H \rightarrow G$ isn't necessarily an inclusion. However, one can always achieve this by replacing the map $f: P \rightarrow N$ by its mapping cylinder. Assume this has been arranged.

As in the previous section, there are pushforward and restriction maps

$$
D_{H} \rightarrow D_{H \subset G} \leftarrow D_{G},
$$

where the first of these is $H$-equivariant and the second is $G$-equivariant. Applying Proposition 3.3, there are an integer $j \geq 0$, a 1-connected finitely dominated based $Y_{H}$ with $H$-action, 1 -connected finitely dominated based $G$-spaces $Y_{H \subset G}$ and $Y_{G}$ and equivariant weak equivalences

$$
\Sigma^{\infty} Y_{H} \simeq \Sigma^{j} D_{H}, \quad \Sigma^{\infty} Y_{H \subset G} \simeq \Sigma^{j} D_{H \subset G} \quad \text { and } \quad \Sigma^{\infty} Y_{G} \simeq \Sigma^{j} D_{G} .
$$

Since $B H$ is a closed Poincaré space, $Y_{H}$ has the unequivariant homotopy type of a sphere.

With respect to these identifications, the pushforward and restriction maps, after $j$-fold suspending, are identified with stable maps

$$
Y_{H} \rightarrow Y_{G \subset H} \leftarrow Y_{G},
$$

where the left map is $H$-equivariant and the right one is $G$-equivariant. We may assume that each of the three spaces above is a equivariant based $\mathrm{CW}$ complex which is free away from the basepoint. Since all of these are equivariantly finitely dominated, a straightforward obstruction theory argument shows that, at the expense of suspending these spaces finitely many times, we can represent the above stable maps by unstable ones (ie we are in effect replacing $Y_{H}, Y_{H \subset G}$ and $Y_{G}$ by their finite $k$-fold suspensions 
for some $k$ sufficiently large.) Therefore, we can assume without loss in generality that the above equivariant stable maps are unstable.

Applying the Borel constructions, we obtain a commutative diagram

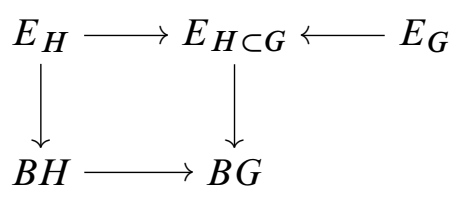

in which $E_{H}=Y_{H} \times_{H} E H$, etc.

Let $T_{H}$ and $T_{G}$ be the mapping cylinders of the maps $E_{H} \rightarrow B H$ and $E G \rightarrow T_{G}$. Then

$$
\left(T_{H}, E_{H}\right) \quad \text { and } \quad\left(T_{G}, E_{G}\right)
$$

are Poincaré pairs of the same dimension [5, p 22].

Proposition 4.1 With respect to the identification $T_{G} \simeq B G$, the diagram (1) is a Poincaré embedding of the map

$$
B H \rightarrow B G \stackrel{\sim}{\rightarrow} T_{G} .
$$

Proof We need to first show that the square

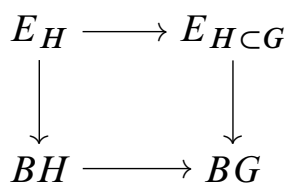

is a homotopy pushout.

To see this, first note that the vertical fibrations of the square come equipped with sections, since they are unreduced Borel constructions of the based spaces $Y_{H}$ and $Y_{H \subset G}$. The homotopy pushout property will follow if we can show that the induced map between the quotient spaces induced by these sections is a weak equivalence.

Now, if $Y$ is a based $G$ space, then the quotient space of the canonical section $B G \rightarrow Y \times_{G} E G$ is just the reduced Borel construction

$$
Y \wedge_{G}(E G)_{+} .
$$

Consequently, we need to show that the map of reduced Borel constructions

$$
Y_{H} \wedge_{H}(E H)_{+} \rightarrow Y_{H \subset G} \wedge_{G}(E G)_{+}
$$


is a weak equivalence. This is a map of 1-connected spaces, so it will be enough to check that the induced map of suspension spectra is a weak equivalence. After desuspending, the map of suspension spectra is identified with the map of homotopy orbit spectra

$$
\left(D_{H}\right)_{h H} \rightarrow\left(D_{H \subset G}\right)_{h G} .
$$

In order to show that the latter map is an equivalence, we use the norm map :

$$
\eta_{H}: D_{H} \wedge_{h H} E \rightarrow E^{h H}
$$

This map was introduced in [5] and was shown to be a weak equivalence for all $E$ whenever $B H$ is a finitely dominated space. The norm map is natural in $E$.

Associated with an inclusion $H \subset G$ there is, more generally, a norm map

$$
\eta_{H \subset G}: D_{H \subset G} \wedge_{h G} E \rightarrow E^{h H}
$$

which is natural in $G$-spectra $E$ (the construction of this more general norm map is virtually identical to the one given in [5] and we will leave these details to the reader). Again this norm map is a weak equivalence whenever $B H$ is finitely dominated.

Furthermore, both kinds of norm maps are compatible with pushforward and restriction in the sense that the evident diagram

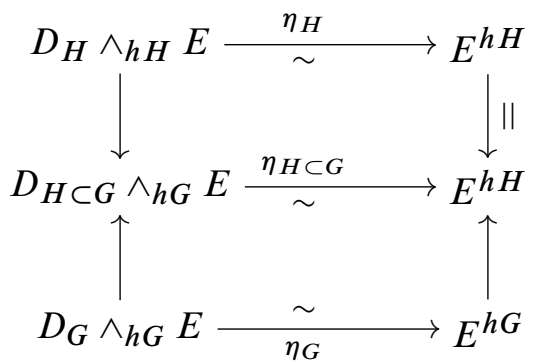

is homotopy commutative. Applying the upper square of the diagram to $E=S^{0}$ (with trivial action) shows that the map

$$
\left(D_{H}\right)_{h H} \rightarrow\left(D_{H \subset G}\right)_{h G}
$$

induced by pushforward is a weak equivalence. We have now shown that (2) is a homotopy pushout.

The second step of the proof is to establish compatibility of the fundamental classes. By $[2,2.3]$, we are only required to show that the fundamental classes of $\left(T_{G}, E_{G}\right)$ and $\left(T_{H}, E_{H}\right)$ are compatible. For this, we need to know how the fundamental classes arise. 
Recall that $E_{G} \subset T_{G}$ is stably identified with $D_{G} \times_{G} E G \rightarrow B G$. Up to a shift in degree, the homology of the quotient $T_{G} / E_{G}$ is the same as the homology of the spectrum given by collapsing the preferred section $B G \rightarrow E_{G} \times_{G} E G$ and this is just the homotopy orbit spectrum $\left(D_{G}\right)_{h G}$. Let

$$
\alpha_{G}: S^{0} \rightarrow\left(D_{G}\right)_{h G}
$$

be the homotopy class which corresponds to the "unit" map

$$
B G_{+} \rightarrow S^{0}
$$

(given unstably by mapping $B G$ to the non-basepoint of $S^{0}$ ) by means of the norm equivalence

$$
\eta_{G}:\left(D_{G}\right)_{h G} \simeq D_{G} \wedge_{h G} S^{0} \stackrel{\sim}{\rightarrow}\left(S^{0}\right)^{h G}=\operatorname{map}\left(B G_{+}, S^{0}\right) .
$$

Then with respect to our identifications, $\alpha_{*}\left(\left[S^{0}\right]\right) \in H_{0}\left(\left(D_{G}\right)_{h G}\right)$ is a fundamental class (cf [5, p 441]).

Compatibility of the fundamental classes is now a direct consequence of the homotopy commutativity of (3) and the observation that the composite $B H_{+} \rightarrow B G_{+} \rightarrow S^{0}$ is the unit map. The completes the proof of Proposition 4.1.

\section{Untwisting}

By Proposition 4.1, the composite

$$
B H \longrightarrow B G \stackrel{\sim}{\longrightarrow} T_{G}
$$

Poincare embeds with diagram (1). Although $T_{G}$ has the homotopy type of $N$, the Poincaré pair $\left(T_{G}, E_{G}\right)$ need not have the homotopy type of $\left(N \times D^{j}, \partial\left(N \times D^{j}\right)\right)$. To obtain a Poincaré embedding into the latter, we will need to twist by an inverse to the Spivak fibration of $N$.

Let $\tau: S(\tau) \rightarrow N$ be a choice of fiber homotopy inverse to the Spivak fibration of $N$. Continuing to identify $N$ with $B G$, we form the fiberwise join

$$
S(\tau) *_{B G} E_{G} \rightarrow B G
$$

To simplify notation denote its total space by ${ }^{\tau} E_{G}$.

Algebraic ${ }^{3} \mathcal{G}$ Geometric Topology, Volume 7 (2007) 
Similarly, applying fiberwise join with $\tau$ to the other terms in the top row of (1) we obtain a modified diagram

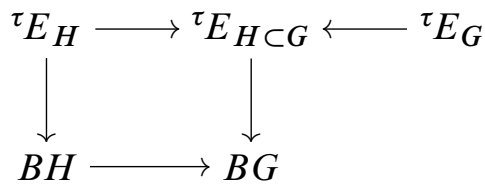

which represents a Poincaré embedding, but this time the ambient Poincare space is $N \times D^{j}$. The proof of Theorem A is now complete in the case when $P$ and $N$ are connected.

\section{The fibered theory}

Recall that $G$ is a topological group model for the loop space $\Omega N$. The unreduced Borel construction of $G$ acting on the dualizing spectrum $D_{G}$ produces a fibered spectrum over $B G$. A more direct construction can be given producing a fibered spectrum over $N$. The latter will enable us to complete the proof of Theorem A in the general case. The new construction is conceptually simpler, albeit more technical.

Definition 5.1 Let $X$ be a space. Let $R(X)$ be the category of retractive spaces over $X$. An object is a space $Y$ equipped with maps $s_{Y}: X \rightarrow Y$ and $r_{Y}: Y \rightarrow X$ such that $r_{Y} \circ s_{Y}$ is the identity map. A morphism $f: Y \rightarrow Z$ is a map of underlying spaces which commutes with their given structure maps: $r_{Z} \circ f=r_{Y}$ and $f \circ s_{Y}=s_{Z}$. A morphism is a weak equivalence if it is a weak homotopy equivalence of underlying spaces. It is a fibration if it is a Serre fibration of underlying spaces. It is a cofibration if it has the left lifting property with the acyclic fibrations (= fibrations which are also weak equivalences). In particular, an object $Y$ is fibrant if $r_{Y}: Y \rightarrow X$ is a Serre fibration. It is cofibrant if $s_{Y}: X \rightarrow Y$ is a Serre cofibration (ie $s_{Y}$ is an inclusion and the pair $(Y, X)$ is a retract of a pair $(Z, X)$ in which $Z$ is obtained from $X$ by attaching cells).

\section{Fibered spectra}

Schwede has shown that the spectra built from objects of any (pointed, simplicial) model category again form a model category. We will apply his result to the model category of retractive spaces $R(X)$.

Given objects $Y, Z \in R(X)$, the hom-set $\operatorname{hom}_{R(X)}(Y, Z)$ may be topologized as a subspace of the function space of all continuous maps $Y \rightarrow Z$ of underlying spaces, 
where the function space is equipped with the compactly generated compact open topology. This gives $R(X)$ the structure of a topological model category. In particular, $R(X)$ is simplicial.

Given an object $Y \in R(X)$, its (reduced) fiberwise suspension is given by

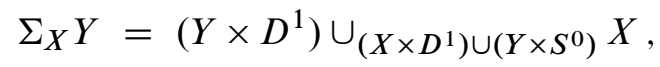

where $\left(X \times D^{1}\right) \cup\left(Y \times S^{0}\right)$ is amalgamated along $X \times S^{0}$ and the map

$$
\left(X \times D^{1}\right) \cup\left(Y \times S^{0}\right) \rightarrow X
$$

is defined as the composite of the inclusion $\left(X \times D^{1}\right) \cup\left(Y \times S^{0}\right) \subset Y \times D^{1}$, followed by first factor projection $Y \times D^{1} \rightarrow Y$, followed by $r_{Y}: Y \rightarrow X$. Then $\Sigma_{X}$ defines an endofunctor of $R(X)$.

Definition 5.2 A fibered spectrum $\mathcal{E}$ over $X$ consists of objects $\mathcal{E}_{j} \in R(X)$ for $j \in \mathbb{N}$ together with (structure) maps

$$
\Sigma_{X} \mathcal{E}_{j} \rightarrow \mathcal{E}_{j+1}
$$

where $\Sigma_{X}: R(X) \rightarrow R(X)$ is the reduced fiberwise suspension functor. A morphism $\mathcal{E} \rightarrow \mathcal{E}^{\prime}$ is given by maps $\mathcal{E}_{j} \rightarrow \mathcal{E}_{j}^{\prime}$ which are compatible with the structure maps.

We say that $\mathcal{E}$ is fibrant if the adjoints to the structure maps are weak homotopy equivalences of underlying spaces. Any fibered spectrum $\mathcal{E}$ can be converted into a fibrant one $\mathcal{E}^{\mathrm{f}}$ in which

$$
\mathcal{E}_{j}^{\mathrm{f}}:=\underset{k}{\operatorname{hocolim}} \Omega_{X}^{k} \mathcal{E}_{j+k},
$$

where the homotopy colimit is taken in $R(X)$, and $\Omega_{X}^{k}$ is the adjoint to $k$-fold reduced fiberwise suspension. The above is called fibrant replacement.

We are now ready to describe the model structure on fibered spectra. A morphism $\mathcal{E} \rightarrow \mathcal{E}^{\prime}$ is a weak equivalence if the associated morphism of fibrant replacements $\mathcal{E}^{\mathrm{f}} \rightarrow\left(\mathcal{E}^{\prime}\right)^{\mathrm{f}}$ is a levelwise weak equivalence: for each $j$, the map $\mathcal{E}_{j}^{\mathrm{f}} \rightarrow\left(\mathcal{E}^{\prime}\right)_{j}^{\mathrm{f}}$ is required to be weak equivalence of $R(X)$. A morphism $\mathcal{E} \rightarrow \mathcal{E}^{\prime}$ is a cofibration if the maps

$$
\mathcal{E}_{0} \rightarrow \mathcal{E}_{0}^{\prime} \quad \text { and } \quad \mathcal{E}_{j} \cup_{\Sigma_{X} \mathcal{E}_{j-1}} \Sigma_{X} \mathcal{E}_{j-1}^{\prime} \rightarrow \mathcal{E}_{j}^{\prime}
$$

are cofibrations of $R(X)$. A morphism is a fibration if it has the right lifting property with respect to the acyclic cofibrations.

Remark 5.3 Several model structures on parametrized spectra are described in the forthcoming book by May and Sigurdsson [10]. This is one of them. 


\section{The fibered spectrum $\mathcal{D}(X)$}

Let $(X, x)$ be a based space and $j \geq 0$ an integer. Then the wedge $X \vee_{x} S^{j}$ is defined and we have a projection

$$
X \vee_{x} S^{j} \rightarrow X
$$

We convert the latter into a Hurewicz fibration by replacing the wedge with the homotopy equivalent space

$$
E(X, x)_{j}:=X^{I} \cup_{P_{x} X} P_{x} X \times S^{j},
$$

where $X^{I}$ is the space of paths $[0,1] \rightarrow X$ and $P_{X} X$ is the subspace of those paths which map 0 to $x$. The inclusion $P_{x} X \rightarrow P_{x} X \times S^{j}$ is given by selecting the basepoint of $S^{j}$. The map $E(X, x)_{j} \rightarrow X$ is induced by evaluating a path at its endpoint. Note that $E(X, x)_{j} \rightarrow X$ has a preferred section given by $x \mapsto c_{x}$, where $c_{x}$ is the constant path having value $x$.

Lemma 5.4 The fiber at $x$ of $E(X, x)_{j} \rightarrow X$ is, up to homotopy, the smash product

$$
\left(\Omega_{x} X\right)+\wedge S^{j}
$$

where $\Omega_{x} X$ is the based loop space of $X$ and $\left(\Omega_{x} X\right)_{+}$is the effect of adding a disjoint basepoint.

Proof The fiber at $x \in X$ is

$$
P_{x} X \cup_{\Omega_{x} X}\left(\Omega_{x} X \times S^{j}\right),
$$

and this has the homotopy type of the quotient

$$
\left(\Omega_{x} X \times S^{j}\right) /\left(\Omega_{x} X \times *\right)=\left(\Omega_{x} X\right)_{+} \wedge S^{j} .
$$

Definition 5.5 Denote the space of sections of $E(X, x)_{j} \rightarrow X$ by

$$
\mathcal{D}(X, x)_{j} .
$$

Note that $\mathcal{D}(X, x)_{j}$ comes equipped with a preferred basepoint.

Define a fibration

$$
\mathcal{D}(X)_{j} \rightarrow X
$$

whose total space is the space of pairs

$$
(x, \sigma),
$$

where $x \in X$ and $\sigma \in D(X, x)_{j}$ (topologize it as a subspace of $X \times \operatorname{map}\left(X, X^{I} \times S^{j}\right)$ ). As each fiber $\mathcal{D}(X, x)_{j}$ is based, $\mathcal{D}(X)_{j} \rightarrow X$ comes equipped with a preferred section. 
The collection

$$
\mathcal{D}(X):=\left\{\mathcal{D}(X)_{j}\right\}_{j}
$$

can be given the structure of a fibered spectrum over $X$. To see this, define an auxiliary space $\mathcal{C}(X)_{j}$ in a way similar to $\mathcal{D}(X)_{j}$, but where we replace the sphere $S^{j}$ by $D^{j+1}$. The standard radial contraction of the disk to a point induces a fiberwise contraction of $\mathcal{C}(X)_{j}$ to the zero object.

Furthermore, there is an evident pushout square of inclusions:

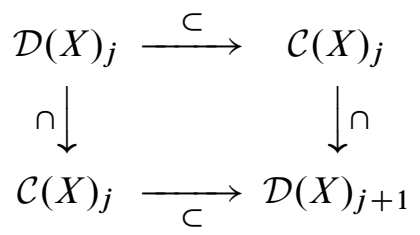

We obtain a map $\Sigma_{X} \mathcal{D}(X)_{j} \rightarrow \mathcal{D}(X)_{j+1}$ using the null homotopies provided by each copy of $\mathcal{C}(X)_{j}$. We infer that $\mathcal{D}(X)$ is a fibered spectrum.

The following result explains the relationship between $\mathcal{D}(X)$ and the dualizing spectrum of the loop space of $X$.

Theorem 5.6 If $X \simeq B G$, then there is a weak equivalence of fibered spectra

$$
\mathcal{D}(X) \simeq E G \times_{G} D_{G} .
$$

Proof The proof will use the following fact: up to fiber homotopy equivalence, a fibration over a connected based space can always be regarded as a Borel construction. Specifically, if $E \rightarrow X$ is a fibration, we identify $X$ with $B G$ for a suitable topological group $G$. The fiber product

$$
E G \times{ }^{B G} E
$$

is then a $G$-space (because it is a $G$-invariant subspace of the product $E G \times E$, where $G$ acts trivially on $E$ ) whose Borel construction recovers $E$ up to fiberwise weak equivalence. Notice that $E G \times{ }^{B G} E$ has the underlying homotopy type of the fiber of $E \rightarrow X$; we call it the thick fiber.

We now begin the proof. Without loss in generality, take $X=B G$. Consider first the fibration

$$
\mathcal{D}(B G)_{0} \rightarrow B G \text {. }
$$

Let $x \in B G$ be a point. Then the fiber at $x$ is the space of sections of the fibration

$$
B G^{I} \cup_{P_{x} B G}\left(P_{x} B G\right) \times S^{0} \rightarrow B G .
$$

The latter has a preferred identification up to homotopy with the fibration

$$
B G \amalg E_{x} G \rightarrow B G,
$$


where $E_{x} G=E G \times{ }_{G} G_{X}$, and $G_{x}$ is the fiber of the universal principal bundle $E G \rightarrow B G$ at $x$.

The space of sections of this last fibration is precisely the homotopy fixed point space

$$
\left(\left(G_{X}\right)_{+}\right)^{h G}=\operatorname{maps}\left(E G,\left(G_{X}\right)_{+}\right)^{G} .
$$

We now compute the thick fiber. The above shows that it is given up to equivariant weak equivalence by the space of pairs

$$
(y, \sigma)
$$

with $y \in E G$ and $\sigma$ a homotopy fixed point of

$$
\left(\left(G_{x}\right)_{+}\right)^{h G},
$$

where $x \in B G$ is the projection of $y$ (ie $y \in G_{x}$ ). By definition of the thick fiber, the action of $G$ on this pair is given by $g \cdot(y, \sigma):=(g y, \sigma)$.

Since $y \in G_{x}$, we have an isomorphism

$$
h_{y}: G \stackrel{\cong}{\rightarrow} G_{x}
$$

given by $g \mapsto g y$. Using this isomorphism, we get a homotopy fixed point

$$
\sigma_{y}=\left(h_{y}\right)^{-1} \circ \sigma \in\left(G_{+}\right)^{h G} \text {. }
$$

The assignment

$$
(y, \sigma) \mapsto \sigma_{y}
$$

defines an equivariant weak equivalence from the thick fiber to $\left(G_{+}\right)^{h G}$. We infer that $\mathcal{D}(B G)_{0}$ is fiber homotopy equivalent to the Borel construction of $G$ acting on $\left(G_{+}\right)^{h G}$.

The case of the fibration

$$
\mathcal{D}(B G)_{j} \rightarrow B G
$$

for $j>0$ is essentially the same, but now the argument gives an equivariant weak equivalence between the thick fiber and $\left(S^{j} \wedge\left(G_{+}\right)\right)^{h G}$. Hence, we have a fiber homotopy equivalence

$$
\mathcal{D}(B G)_{j} \simeq E G \times_{G}\left(S^{j} \wedge\left(G_{+}\right)\right)^{h G} .
$$

We leave it to the reader to verify that the equivalences for each $j$ that we produced give a morphism of fibered spectra. We have now shown that the fibered spectrum $\mathcal{D}(B G)$ is identified with the fibered spectrum whose $j$-th term is $E G \times_{G}\left(S^{j} \wedge\left(G_{+}\right)\right)^{h G}$. To complete the proof, we identify this last fibered spectrum with the unreduced Borel construction of $D_{G}$.

Algebraic 83 Geometric Topology, Volume 7 (2007) 
This follows immediately from the fact that the evident map of spaces

$$
E G \times_{G}\left(S^{j} \wedge\left(G_{+}\right)\right)^{h G} \rightarrow E G \times_{G} Q\left(S^{j} \wedge\left(G_{+}\right)\right)^{h G}
$$

is $(2 j-c)$-connected, for some constant $c \geq 0$ independent of $j$ (this uses the Freudenthal suspension theorem and the assumption of $X=B G$ being finitely dominated). A map of such fibered spectra is clearly a weak equivalence.

Remark 5.7 If $X=\amalg_{\alpha} X_{\alpha}$ is a space decomposed into connected components, then we have a decomposition of the fibered spectrum

$$
\mathcal{D}(X)=\coprod_{\alpha} \mathcal{D}\left(X_{\alpha}\right) .
$$

In particular, if $X$ is a Poincaré space, the above theorem shows that $\mathcal{D}(X) \rightarrow X$ is the stable Spivak fibration.

\section{Extension to maps}

Given a map of spaces

$$
A \rightarrow X
$$

we can associate a fibration

$$
\mathcal{D}(A \rightarrow X)_{j} \rightarrow X
$$

whose total space consists of pairs

$$
(x, \sigma)
$$

in which $x \in X$ an $\sigma$ is a section of $E(X, x)_{j} \rightarrow X$ along $A$, ie $\sigma$ is a section of the associated pullback fibration. The collection

$$
\mathcal{D}(A \rightarrow X):=\left\{\mathcal{D}(A \rightarrow X)_{j}\right\}_{j \geq 0},
$$

forms a fibered spectrum over $X$. The case of the identity map $X \rightarrow X$ recovers $\mathcal{D}(X)$.

If $f: A \rightarrow B$ is a map of spaces over $X$, then we obtain a restriction map

$$
f^{!}: \mathcal{D}(B \rightarrow X) \rightarrow \mathcal{D}(A \rightarrow X)
$$

This is a morphism of fibered spectra over $X$.

There is also a pushforward map

$$
f_{!}: \mathcal{D}(A \rightarrow B) \rightarrow \mathcal{D}(A \rightarrow X)
$$

which is a map of fibered spectra covering $B \rightarrow X$.

Algebraic ${ }^{3} \mathcal{G}$ Geometric Topology, Volume 7 (2007) 


\section{Proof of Theorem $A$ in the general case}

We apply the constructions of the last section to $f: P \rightarrow N$ to get a diagram:

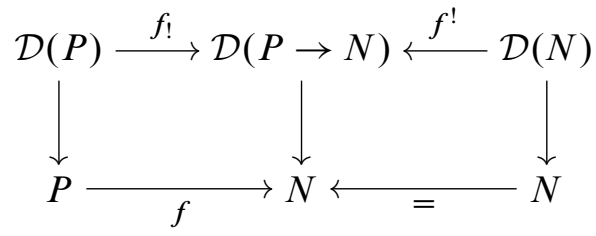

By analogy with the connected case, each of the fibered spectra appearing above is a fibered suspension spectrum (we omit the details).

The rest of the proof is as in the connected case: the above diagram fiberwise suspends to an associated commutative diagram of spaces

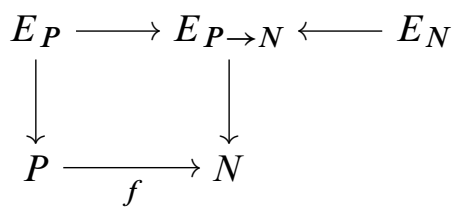

which is a Poincare embedding into the mapping cylinder of $E_{N} \rightarrow N$. We must then twist if necessary by a suitable element in the Grothendieck group of spherical fibrations over $N$ to get a Poincaré embedding into $N \times D^{j}$ for suitable $j$. The proof of Theorem A is now complete.

\section{Proof of Theorem B}

We will prove uniqueness when $N$ is closed and has trivial Spivak fibration. The general case, which follows by a twisting argument similar to that of the last section, is left to the reader.

Suppose we are given two Poincaré embeddings of $f_{j}: P \rightarrow N \times D^{j}$, with diagrams

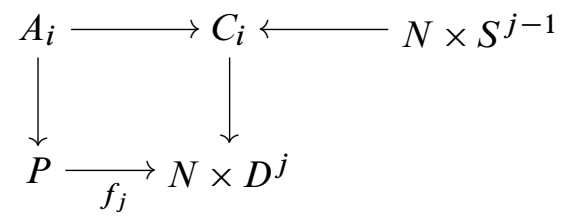

for $i=0,1$. The assumptions on $N$ imply that $A_{i} \rightarrow P$ are Spivak fibrations for $P$. 
To minimize technical difficulties, it is more convenient to replace $N \times D^{j}$ by $N$ (by means of first factor projection), thereby rewriting each diagram for $i=0,1$ as:

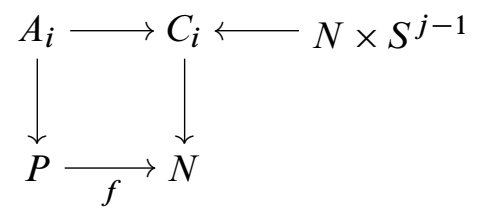

There is no loss of information, since we can recover the original diagram up to concordance by taking suitable mapping cylinders.

If $j$ is large, the Spivak fibration is unique up to fiber homotopy equivalence. Hence there is a fiber homotopy equivalence over $P$

$$
A_{0} \simeq A_{1}
$$

Therefore, without loss in generality, we can assume $A_{0}=A_{1}$. To simplify notation, we set

$$
A:=A_{0} .
$$

The next step is to relate $C_{0}$ with $C_{1}$. We first make some definitions.

Definition 7.1 Let $T(N)$ be the category whose objects are spaces $Y$ equipped with a map $Y \rightarrow N$. A morphism is a map compatible with the structure map to $N$. Thus $T(N)$ is the category of spaces over $N$. A morphism is a weak equivalence when the underlying map of spaces is a weak homotopy equivalence.

The unreduced fiberwise suspension of $Y$ is defined to be

$$
S_{N} Y:=\left(Y \times D^{1}\right) \cup_{Y \times S^{0}}\left(N \times S^{0}\right) .
$$

It is an object of $T\left(N \times D^{1}\right)$ but we usually regard it as an object of $T(N)$ using the first factor projection $N \times D^{1} \rightarrow N$.

Definition 7.2 Given a Poincaré embedding

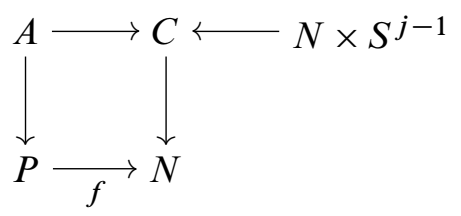

Algebraic 83 Geometric Topology, Volume 7 (2007) 
its decompression is the associated Poincaré embedding

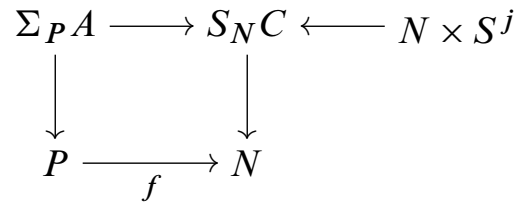

where $N \times S^{j} \rightarrow S_{N} C$ is the fiberwise suspension of $N \times S^{j-1} \rightarrow C$.

Definition 7.3 Let $A \rightarrow X$ be an inclusion map of $T(N)$. The fiberwise quotient is

$$
X / / A:=X \cup_{A} N
$$

which is an object of the retractive space category $R(N)$, but can also be regarded as an object of $T(N)$ by means of the forgetful functor $R(N) \rightarrow T(N)$.

To see that $S_{N} C_{0}$ and $S_{N} C_{1}$ are weak equivalent, we compare them both to $P / / A$ by means of the "excision" weak equivalences

$$
P / / A:=P \cup_{A} N \stackrel{\sim}{\longrightarrow} N \cup_{C_{i}} N \simeq S_{N} C_{i}
$$

This produces the desired chain of weak equivalences.

The final step in the proof of uniqueness is to consider the maps

$$
N \times S^{j} \rightarrow S_{N} C_{i}
$$

appearing in the decompressions of our given Poincaré embeddings. Call these maps $\alpha_{i}$. We must show that our identification $S_{N} C_{0} \simeq S_{N} C_{1}$ is compatible with $\alpha_{i}$ up to homotopy.

This identification arises by identifying each $S_{N} C_{i}$ with $P / / A$. So the $\alpha_{i}$ correspond to a pair of maps

$$
\beta_{i}: N \times S^{j} \rightarrow P / / A
$$

which we need to prove are fiberwise homotopic. An unraveling of the definitions shows that these are the fiberwise collapse maps of the two Poincaré embeddings (see Section 8).

Each one of these maps is therefore fiberwise dual to the map

$$
f^{+}: P^{+} \rightarrow N^{+}
$$

(again, see Section 8). By the uniqueness of fiberwise duality maps, it follows that $\beta_{0}$ and $\beta_{1}$ are homotopic when $j \gg 0$. This completes the proof of uniqueness.

Algebraic $6 \mathcal{G}$ Geometric $\mathcal{T}$ opology, Volume 7 (2007) 


\section{Appendix: fiberwise duality}

Suppose $N$ is a Poincaré duality space, possibly with boundary $\partial N$. Let $X$ and $Y$ be objects of $R(N)$. The fiberwise smash product $X \wedge_{N} Y$ of $X$ and $Y$ is the object

$$
X \times_{N} Y \cup_{X \cup_{N} Y} N,
$$

where $X \times{ }_{N} Y$ is the fiber product of $X$ and $Y$ along $N$. The map $X \cup_{N} Y \rightarrow X \times{ }_{N} Y$ is defined using the structure maps $N \rightarrow X$ and $N \rightarrow Y$.

Remark 8.1 As usual, in order to get a homotopy invariant theory, we must replace the fiberwise smash product with its derived version, ie we replace $X$ and $Y$ by their fibrant/cofibrant approximations. In what follows, we will be suppressing this aspect from the notation. The reader is forewarned.

Denote the double of $N$ by

$$
N^{+}:=N / / \partial N \text {. }
$$

This is an object of $R(N)$ whose underlying space is $N \cup_{\partial N} N$. Here are some special cases:

(1) If $\partial N$ is empty, then $N^{+}$is just $N \times S^{0}$.

(2) The $j$-fold unreduced fiberwise suspension $S_{N}^{j} N^{+}$coincides with $\left(N \times D^{j}\right)^{+}$. In particular, if $\partial N$ is empty, we get $N \times S^{j}$.

A duality map for $X$ and $Y$ is a morphism

$$
d: N^{+} \rightarrow X \wedge_{N} Y
$$

such that for all (cofibrant and fibrant) fibered spectra $\mathcal{E}$, the operation taking $g$ to $\left(g \wedge_{N} \mathrm{id}_{Y}\right) \circ d$ induces an isomorphism of abelian groups

$$
[X, \mathcal{E}]_{N} \cong\left[N^{+}, \mathcal{E} \wedge_{N} Y\right]_{N},
$$

where $[,]_{N}$ means fiberwise homotopy classes, and the fiberwise smash product $\mathcal{E} \wedge_{N} Y$ is defined by $\left(\mathcal{E} \wedge_{N} Y\right)_{j}=\mathcal{E}_{j} \wedge_{N} Y$.

Remarks 8.2 (1) When $N=D^{n}$ is a disk, fiberwise duality amounts to ordinary Spanier-Whitehead $n$-duality.

(2) When $N$ is an arbitrary space, a closely related type of fiberwise duality theory was considered by Vogell [14], who considered maps of $R(N \times N)$ of the form

$$
X \sharp Y \rightarrow T_{j},
$$

Algebraic 83 Geometric Topology, Volume 7 (2007) 
where $\sharp$ denotes the external fiberwise smash product of $X$ and $Y$ (this is retractive over $N \times N$ ), and $T_{j}$ is given as follows: consider $N$ as a space over $N \times N$ via the diagonal and make it retractive by adding a disjoint copy of $N \times N$. Call the resulting object $T_{0}$. Then we take $T_{j}$ to be the $j$-fold fiberwise suspension of $T_{0}$ (in Vogell's terminology, $T_{j}$ is the fiberwise Thom complex of the rank $j$-trivial bundle). If $N$ is a closed Poincare space, then Vogell's duality maps are related to Richter's via a certain umkehr correspondence. The theories in this case are equivalent.

We now state without proof some basic properties of fiberwise duality maps.

- (Suspension) If $d$ is a fiberwise duality map, so is its fiberwise suspension

$$
\Sigma_{N} d:\left(N \times D^{1}\right)^{+} \rightarrow X \wedge_{N} \Sigma_{N} Y .
$$

- (Switching) If $d$ is a fiberwise duality map, so is the composite

$$
N^{+} \stackrel{d}{\longrightarrow} X \wedge_{N} Y \stackrel{\text { twist }}{\longrightarrow} Y \wedge_{N} X .
$$

- (Existence) Given a finitely dominated object $X$, there is always a finitely dominated object $Y$, an integer $j \geq 0$ and a duality map

$$
\left(N \times D^{j}\right)^{+} \rightarrow X \wedge_{N} Y .
$$

- (Uniqueness) Without loss in generality take $Y$ to be both fibrant and cofibrant. Let $d^{\prime}: N^{+} \rightarrow X \wedge \wedge_{N} Z$ be another fiberwise duality map with $Z$ cofibrant and fibrant. Assume $j$ is large. Then there is a weak equivalence $h: \Sigma_{N}^{j} Y \stackrel{\sim}{\rightarrow} \Sigma_{N}^{j} Z$ such that the composite

$$
\left(N \times D^{j}\right)^{+} \stackrel{\Sigma_{N}^{j} d}{\longrightarrow} X \wedge_{N} \Sigma_{N}^{j} Y \stackrel{\mathrm{id} \wedge_{N} h}{\longrightarrow} X \wedge_{N} \Sigma_{N}^{j} Z
$$

has the same fiberwise homotopy class as $S_{N}^{j} d^{\prime}$.

Geometrically, fiberwise duality maps arise from Poincaré embeddings as follows. Suppose we are given a Poincaré embedding:

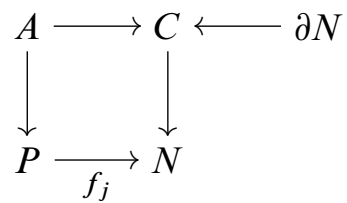

Then there is a fiberwise duality map

$$
d: N^{+} \rightarrow P^{+} \wedge_{N} P / / A
$$

Algebraic 83 Geometric Topology, Volume 7 (2007) 
which may be defined as a composition of two maps: the fiberwise collapse

$$
N^{+} \rightarrow P / / A
$$

followed by the fiberwise diagonal

$$
P / / A \rightarrow P^{+} \wedge_{N} P / / A
$$

where $P^{+}$denotes $P \amalg N$.

As a fiberwise homotopy class, the collapse arises from the chain of fiberwise pairs

$$
(N, \partial N) \stackrel{\sim}{\longleftarrow}\left(P \cup_{A} C, \partial N\right) \longrightarrow\left(P \cup_{A} N, N\right)
$$

by taking fiberwise quotients. Similarly, the fiberwise diagonal arises from the evident fiberwise diagonal map of pairs

$$
(P, A) \longrightarrow\left(P \times_{N} P, P \times_{N} A\right)
$$

by taking fiberwise quotients.

The following result is due to Richter (unpublished; see [14, p 163] for a dual version in the manifold case).

Proposition 8.3 (Richter Duality) The map

$$
d: N^{+} \rightarrow P^{+} \wedge_{N} P / / A
$$

is a fiberwise duality.

Assume now that $\partial N=\varnothing$ and we are given a Poincaré embedding:

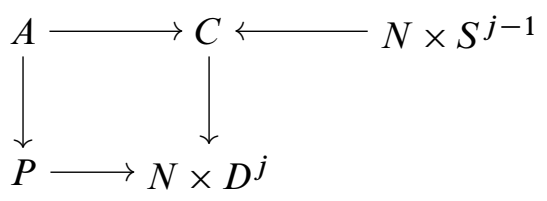

Then the proposition applied in this case with $\mathcal{E}=\Sigma_{N}^{\infty} N^{+}$(the fiberwise suspension spectrum of $N^{+}$) shows that (the stable fiberwise homotopy class of) the map

$$
f^{+}: P^{+} \rightarrow N^{+}
$$

corresponds under the duality isomorphism to the fiberwise collapse

$$
N \times S^{j}=\left(N \times D^{j}\right)^{+} \rightarrow P / / A .
$$

This is the fact we are using in the proof of Theorem B.

Algebraic ${ }^{3} \mathcal{G}$ Geometric Topology, Volume 7 (2007) 


\section{References}

[1] T Bauer, N Kitchloo, D Notbohm, E K Pedersen, Finite loop spaces are manifolds, Acta Math. 192 (2004) 5-31 MR2079597

[2] J R Klein, Poincaré duality embeddings and fiberwise homotopy theory, Topology 38 (1999) 597-620 MR1670412

[3] J R Klein, Poincaré immersions, Forum Math. 11 (1999) 717-734 MR1725594

[4] J R Klein, Poincaré duality spaces, from: "Surveys on surgery theory, Vol. 1", Ann. of Math. Stud. 145, Princeton Univ. Press, Princeton, NJ (2000) 135-165 MR1747534

[5] J R Klein, The dualizing spectrum of a topological group, Math. Ann. 319 (2001) 421-456 MR1819876

[6] J R Klein, Embedding, compression and fiberwise homotopy theory, Algebr. Geom. Topol. 2 (2002) 311-336 MR1917055

[7] J R Klein, Poincaré duality embeddings and fibrewise homotopy theory. II, Q. J. Math. 53 (2002) 319-335 MR1930266

[8] P Lambrechts, D Stanley, Algebraic models of Poincaré embeddings, Algebr. Geom. Topol. 5 (2005) 135-182 MR2135550

[9] P Lambrechts, D Stanley, L Vandembroucq, Embeddings up to homotopy of two-cones in Euclidean space, Trans. Amer. Math. Soc. 354 (2002) 3973-4013 MR1926862

[10] J May, J Sigurdsson, Parametrized homotopy theory arXiv:math. AT/0411656

[11] W Richter, A homotopy-theoretic proof of Williams's metastable Poincaré embedding theorem, Duke Math. J. 88 (1997) 435-447 MR1455528

[12] S Schwede, Spectra in model categories and applications to the algebraic cotangent complex, J. Pure Appl. Algebra 120 (1997) 77-104 MR1466099

[13] M Spivak, Spaces satisfying Poincaré duality, Topology 6 (1967) 77-101 MR0214071

[14] W Vogell, The canonical involution on the algebraic $K$-theory of spaces, from: "Algebraic topology, Aarhus 1982 (Aarhus, 1982)", Lecture Notes in Math. 1051, Springer, Berlin (1984) 156-172 MR764578

[15] C T C Wall, Poincaré complexes. I, Ann. of Math. (2) 86 (1967) 213-245 MR0217791

[16] C T C Wall, Surgery on compact manifolds, second edition, Mathematical Surveys and Monographs 69, American Mathematical Society, Providence, RI (1999) MR1687388Edited and with a foreword by A. A. Ranicki

[17] B Williams, Applications of unstable normal invariants. I, Compositio Math. 38 (1979) 55-66 MR523263

Algebraic $6 \mathcal{G}$ Geometric Topology, Volume 7 (2007) 
[18] B Williams, Hopf invariants, localization and embeddings of Poincaré complexes, Pacific J. Math. 84 (1979) 217-224 MR559639

Wayne State University

Detroit, MI 48202

klein@math. wayne.edu

Received: 30 September 2006

Algebraic 83 Geometric Topology, Volume 7 (2007) 